University of Wollongong

Research Online

Australian Institute for Innovative Materials -

Papers

Australian Institute for Innovative Materials

$1-1-2019$

Intercalation Pseudocapacitance Boosting Ultrafast Sodium Storage in

Prussian Blue Analogs

Baoqi Wang

Zhejiang University

Shuangyu Liu

Global Energy Interconnection Research Institute Co. Ltd

Wenping Sun

University of Wollongong, wenping@uow.edu.au

Yuxin Tang

University of Macau

Hongge Pan

Zhejiang University

See next page for additional authors

Follow this and additional works at: https://ro.uow.edu.au/aiimpapers

Part of the Engineering Commons, and the Physical Sciences and Mathematics Commons

Research Online is the open access institutional repository for the University of Wollongong. For further information contact the UOW Library: research-pubs@uow.edu.au 


\title{
Intercalation Pseudocapacitance Boosting Ultrafast Sodium Storage in Prussian Blue Analogs
}

\author{
Abstract \\ Great expectation is placed on sodium-ion batteries with high rate capability to satisfy multiple \\ requirements in large-scale energy storage systems. However, the large ionic radius and high mass of $\mathrm{Na}$ \\ + hamper its kinetics in the case of diffusion-controlled mechanisms in conventional electrodes. In this \\ study, a unique intercalation pseudocapacitance has been demonstrated in low-vacancy copper \\ hexacyanoferrate, achieving outstanding rate capability. The minimization of the $[\mathrm{Fe}(\mathrm{CN}) 6]$ vacancy \\ enables unhindered diffusion pathways for $\mathrm{Na}+$ and little structural change during the $\mathrm{Fe} 2+/ \mathrm{Fe} 3+$ redox \\ reaction, eliminating solid-state diffusion limits. Moreover, the $\mathrm{Cu}+/ \mathrm{Cu} 2+$ couple is unexpectedly \\ activated, realizing a record capacity for copper hexacyanoferrate. A capacity of $86 \mathrm{mAh} \mathrm{g-1}$ is obtained \\ at $1 \mathrm{C}$, of which $50 \%$ is maintained under $100 \mathrm{C}$ and $70 \%$ is achieved at $0{ }^{\circ} \mathrm{C}$. Such intercalation \\ pseudocapacitance might shed light on exploiting high-rate electrodes among Prussian blue analogs for \\ advanced sodium-ion batteries. \\ Disciplines \\ Engineering | Physical Sciences and Mathematics

\section{Publication Details} \\ Wang, B., Liu, S., Sun, W., Tang, Y., Pan, H., Yan, M. \& Jiang, Y. (2019). Intercalation Pseudocapacitance \\ Boosting Ultrafast Sodium Storage in Prussian Blue Analogs. ChemSusChem: chemistry and \\ sustainability, energy and materials, 12 (11), 2415-2420.

\section{Authors} \\ Baoqi Wang, Shuangyu Liu, Wenping Sun, Yuxin Tang, Hongge Pan, Mi Yan, and Yinzhu Jiang
}




\title{
Intercalation pseudocapacitance boosting ultrafast sodium storage in
}

\section{Prussian blue analogues}

\author{
Baoqi Wang, ${ }^{[a]}$ Shuangyu Liu, ${ }^{[b]}$ Wenping Sun, ${ }^{[c]}$ Yuxin Tang,,${ }^{[d]}$ Hongge Pan,,${ }^{[a]}$ Mi Yan, ${ }^{[a]}$ and Yinzhu \\ Jiang*[a]
}

\begin{abstract}
Great expectation is placed on sodium ion batteries with high rate capability to satisfy multiple requirements in large-scale energy storage systems. However, the large ion radius and high mass of $\mathrm{Na}^{+}$hamper its kinetics in case of diffusion-controlled mechanism in conventional electrode. Herein, a unique intercalation pseudocapacitance is demonstrated in low-vacancy copper hexacyanoferrate, achieving outstanding rate capability. The minimization of $\left[\mathrm{Fe}(\mathrm{CN})_{6}\right]$ vacancy enables unhindered diffusion pathway for $\mathrm{Na}^{+}$and little structural change during $\mathrm{Fe}^{2+} / \mathrm{Fe}^{3+}$ redox reaction, eliminating solid-state diffusion limits. Moreover, the $\mathrm{Cu}^{+} / \mathrm{Cu}^{2+}$ couple is unexpectedly activated realizing a record capacity of copper hexacyanoferrate. A capacity of $86 \mathrm{mAh} \mathrm{g}^{-1}$ is obtained at 1 $\mathrm{C}$, of which $50 \%$ is maintained under $100 \mathrm{C}$ and $70 \%$ is achieved at $0^{\circ} \mathrm{C}$. Such intercalation pseudocapacitance might shed light on exploiting high rate electrodes among Prussian blue analogues for advanced sodium ion batteries.
\end{abstract}

\section{Introduction}

Lithium ion batteries (LIBs) have become the most used secondary battery since its commercialization in early 1990s. ${ }^{[1]}$ With the introduction of LIBs into fields of electric vehicles (EVs) and large-scale energy storage systems (ESSs), the rapid growing demand for LIBs is causing battery components such as lithium and cobalt to skyrocket in price. ${ }^{[2,3]}$ To mitigate the resource shortage and rising cost of lithium, substantial efforts have been devoted to developing "beyond lithium" batteries, where sodium ion batteries (SIBs) attracted immense interests owing to the resource abundance and low prices of sodium..$^{[1,4,5]}$ Considering the higher redox potential of $\mathrm{Na} / \mathrm{Na}^{+}$and heavier

[a] B. Wang, Prof H. Pan, Prof M. Yan, Prof Y. Jiang State Key Laboratory of Silicon Materials, Key Laboratory of Novel Materials for Information Technology of Zhejiang Province and School of Materials Science and Engineering

Zhejiang University

Hangzhou 310027, China

E-mail: yzjiang@zju.edu.cn

[b] Dr S. Liu

State Key Laboratory of Advanced Transmission Technology

Global Energy Interconnection Research Institute Co. Ltd

Beijing 102211, China

[c] DrW. Sun

Institute for Superconducting and Electronic Materials

University of Wollongong

Wollongong NSW 2522, Australia

[d] Prof Y. Tang

Institute of Applied Physics and Materials Engineering University of Macau

Macau, China

Supporting information for this article is given via a link at the end of the document. mass of sodium as compared to those of lithium, SIBs are considered as a promising candidate for the applications in large scale ESSs and smart grids where the energy density becomes less critical. ${ }^{[6]}$ On the other hand, superior electrochemical kinetics is greatly desired for SIBs to adapt for the geographical and climatic complexity in renewable energy storage, while frequency regulation and peak shaving in smart grids demand for high rate capability of SIBs. ${ }^{[3,4]}$

The kinetics and rate capability of a full battery are largely determined by the electrode of a battery, particularly the cathode. Three classes of inorganic cathode materials have been investigated for SIBs including layered metal oxides, ${ }^{[4,}$ 7] polyanionic compounds ${ }^{[8]}$ and Prussian blue analogues (PBAs). Among them, three-dimensional open framework of PBAs are characterized by the weak interaction between the $\mathrm{Na}^{+}$and the anion $p_{\pi}$ electrons. ${ }^{[9,10]}$ This framework offers the possibility for ultrafast sodium kinetics through "intercalation pseudocapacitance", which is featured by fast intercalation behavior with no limitations from solid-state diffusion. Dunn et

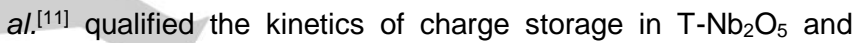
defined that facile ion transport pathway and little structural change in a crystalline network is necessary for intercalation pseudocapacitance. Yang et al. ${ }^{[12]}$ demonstrated an intercalation pseudocapacitance behavior and ultrafast $\mathrm{Zn}^{2+}$ insertion in $\mathrm{VO}_{2}$ nanofibers, achieving the high rate capability $\left(171 \mathrm{mAh} \mathrm{g}^{-1}\right.$ at 300 C) for aqueous zinc-ion batteries. The principle benefit realized from intercalation pseudocapacitance is that high capacity and fast kinetics of electrode can be achieved simultaneously, leading to high rate performance without sacrificing energy density. ${ }^{[11,13]}$ Unfortunately, there is still a lack of studies on intercalation pseudocapacitance of PBAs. This unfulfilled pseudocapacitive behavior might be caused by the imperfection of the framework originated from the coordinated water and vacancies during synthesis.

Herein, we demonstrate intercalation pseudocapacitive behavior in a low-vacancy copper hexacyanoferrate (LV-CuHCF) electrode synthesized in a dehydrated environment, leading to ultrafast sodium kinetics. Benefiting from the rigid highly crystallized framework, both remarkable high specific capacity and outstanding rate capability are achieved. The specific capacity reached $86 \mathrm{mAh} \mathrm{g}^{-1}$ at $1 \mathrm{C}$ rate $\left(1 \mathrm{C}=80 \mathrm{~mA} \mathrm{~g}^{-1}\right)$, of which an unexpected contribution was made by the redox couple of $\mathrm{Cu}^{+} / \mathrm{Cu}^{2+}$. Such capacity is among the highest values of CuHCF-based cathodes for SIBs. ${ }^{[14,15,16]}$ Referring to rate capability, $50 \%$ capacity of the as-prepared CuHCF was maintained even under an extremely high rate of $100 \mathrm{C}$ at room temperature, due to the unique intercalation pseudocapacitance. Furthermore, the CuHCF electrode remained its outstanding rate performance even when tested at freezing point. 


\section{Results and Discussion}

In a perfect PBA framework, the octahedral metal centers are coordinated with $\mathrm{C} \equiv \mathrm{N}^{-}$bridges by $\mathrm{N}$ and $\mathrm{C}$ atoms alternatively forming three-dimensional network as illustrated in Figure 1a. ${ }^{[9,17}$, 18] However, in general synthesis process, the fast precipitation rate of PBAs always accompanies with the generation of $\left[\mathrm{Fe}(\mathrm{CN})_{6}\right]$ vacancies. ${ }^{[17,19]}$ Consequently, coordinated water from the hydrous preparation environment occupies the six corners of the resulting octahedral cavities as seen in Figure 1c. Because $\mathrm{Cu}$ has the largest hydration energy among the first period transition metals, it is easier to coordinate with water molecules in aqueous solution to form hydrated $\mathrm{Cu}^{2+}$ ions. The hydrated $\mathrm{Cu}^{2+}$ ions will bring a lot of water molecules into the CuHCF framework during its growth. Because of the coordinated water, the $\mathrm{Cu}^{2+}$ ions can no longer coordinate with $\mathrm{N}$ atoms of $\left[\mathrm{Fe}(\mathrm{CN})_{6}\right]$ which they are supposed to, leading to $\left[\mathrm{Fe}(\mathrm{CN})_{6}\right]$ vacancies in the framework, eg. $\mathrm{K}_{0.71} \mathrm{Cu}\left[\mathrm{Fe}(\mathrm{CN})_{6}\right]_{0.72} \cdot 3.7 \mathrm{H}_{2} \mathrm{O}^{[16]}$. As a result, the active redox sites are reduced in such high vacancy CuHCF (HV-CuHCF). On the other hand, sodium migration also relies on the integrity of the framework since the ion diffusion in PBA framework is calculated to be a W-shaped path from one face center to another. Once $\left[\mathrm{Fe}(\mathrm{CN})_{6}\right]$ vacancies emerge, the ion pathways become discontinuous, which would deteriorate the sodium intercalation/deintercalation. To minimize the formation of $\left[\mathrm{Fe}(\mathrm{CN})_{6}\right]$ vacancy during the synthesis of $\mathrm{CuHCF}$, a dehydrated synthesis method was designed. Ethanol was used as solvent to prevent $\mathrm{Cu}^{2+}$ ions from coordinating with water. Much less coordinated water is introduced into the CuHCF framework during the precipitation process, resulting in the birth of a low vacancy CuHCF (LV-CuHCF) with a high Fe/Cu ratio of 0.931. In contrast, HV-CuHCF prepared in a hydrous condition shows a low $\mathrm{Fe} / \mathrm{Cu}$ ratio of 0.647 . Figure $1 \mathrm{a}$ and $1 \mathrm{c}$ show the $\mathrm{X}$-ray diffraction (XRD) patterns of LV-CuHCF and HV-CuHCF, both of which can be well indexed to the same cubic structure (JCPDS No. 86-0513). However, there is obvious shift of peaks to lower angle in LVCuHCF, indicating a larger lattice parameter due to the minimization of $\left[\mathrm{Fe}(\mathrm{CN})_{6}\right]$ vacancy that will be favorable for sodium diffusion. As a result, LV-CuHCF is expected to provide more sodium storage sites and unbroken sodium ion pathway, enabling intercalation pseudocapacitance to achieve higher capacity and faster kinetics.

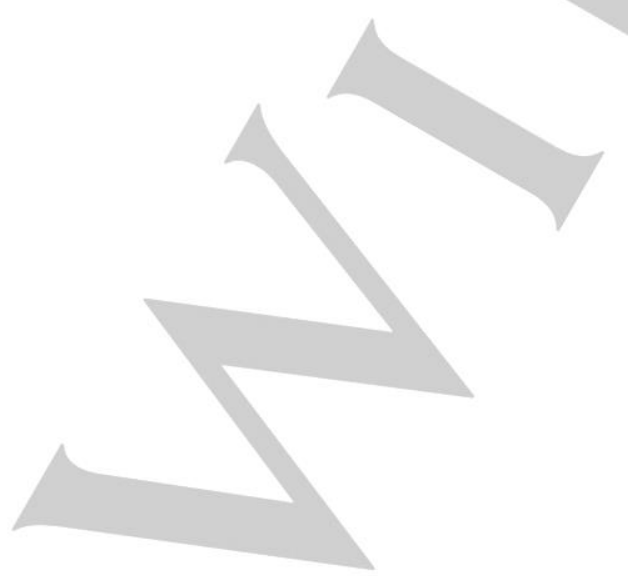

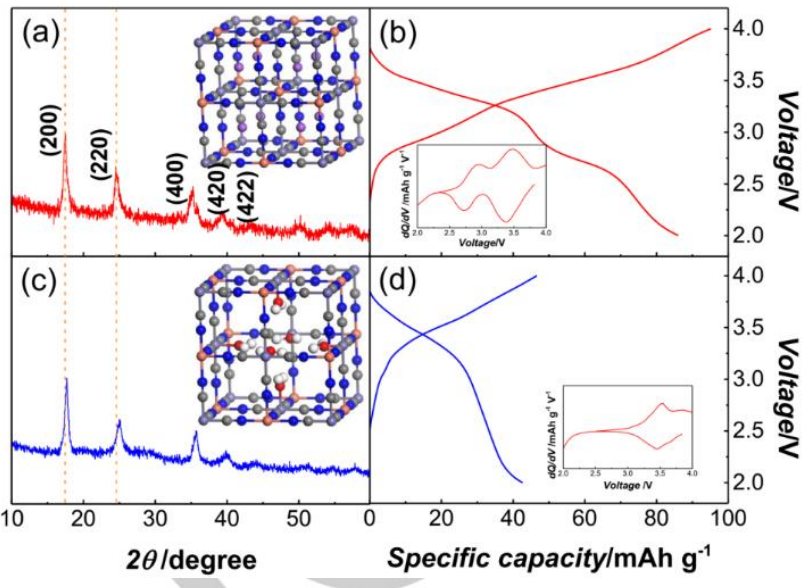

Figure 1. (a) XRD patterns of (a) LV-CuHCF and (c) HV-CuHCF. The illustrations are crystal structures of LV-CuHCF and HV-CuHCF, accordingly. Charge-discharge profiles of (b) LV-CuHCF and (d) HV-CuHCF. Rate: $1 \mathrm{C}$. The derivative curves $(\mathrm{dQ} / \mathrm{dV})$ plotted as a function of $\mathrm{V}$ are shown as inserts.

Considering the low electronic conductivity of PBAs, Ketjen black (KB) was introduced to obtain PBA-carbon composites for both LV-CuHCF and HV-CuHCF. Figure $1 \mathrm{~b}$ and $1 \mathrm{~d}$ compare the first-cycle charge/discharge profiles of LV-CuHCF and HVCuHCF at $1 \mathrm{C}$. As expected, LV-CuHCF exhibits twice more of the capacity of HV-CuHCF, achieving a high record value of 86 mAh g-1 among all previous reports on CuHCF-based cathode materials. In addition to the higher charge/discharge voltage plateau at $3.48 / 3.38 \mathrm{~V}$, an extra plateau at lower voltage of 2.96/2.72 V appears as compared to HV-CuHCF. Such unforeseen pair of plateaus can be ascribed to the redox reaction of copper, which will be discussed later.

Rate capability is one of the primary hurdles that hinders the application of SIBs considering the large ion radius and high mass of sodium. As a unique mechanism, intercalation pseudocapacitance is supposed to be powerful in accelerating sodium kinetics. Figure $2 \mathrm{a}$ and $2 \mathrm{~b}$ display the charge-discharge profiles of LV-CuHCF from 1 to $100 \mathrm{C}$ and HV-CuHCF from 1 to $50 \mathrm{C}$. Upon increasing the current rate to 2, 5, 10, 20 and $50 \mathrm{C}$, the discharge capacity of LV-CuHCF maintains $77,71,64,58$ and $50 \mathrm{mAh} \mathrm{g}^{-1}$. In contrast, HV-CuHCF only possesses a capacity of $17 \mathrm{mAh} \mathrm{g}^{-1}$ at $50 \mathrm{C}$. Impressively, even when cycled at an extremely high rate of $100 \mathrm{C}$ that is the highest rate ever reported for PBAs, the LV-CuHCF electrode can still exhibit a reversible capacity of $41 \mathrm{mAh} \mathrm{g}^{-1}$, corresponding to a high capacity retention of $\sim 50 \%$ of the capacity at $1 \mathrm{C}$. Besides the appealing rate capability, the long cycle life is also demonstrated in the LVCuHCF electrode with a reversible capacity of $42 \mathrm{~mA} \mathrm{~h} \mathrm{~g}^{-1}$ at 10 C over 1000 cycles. 

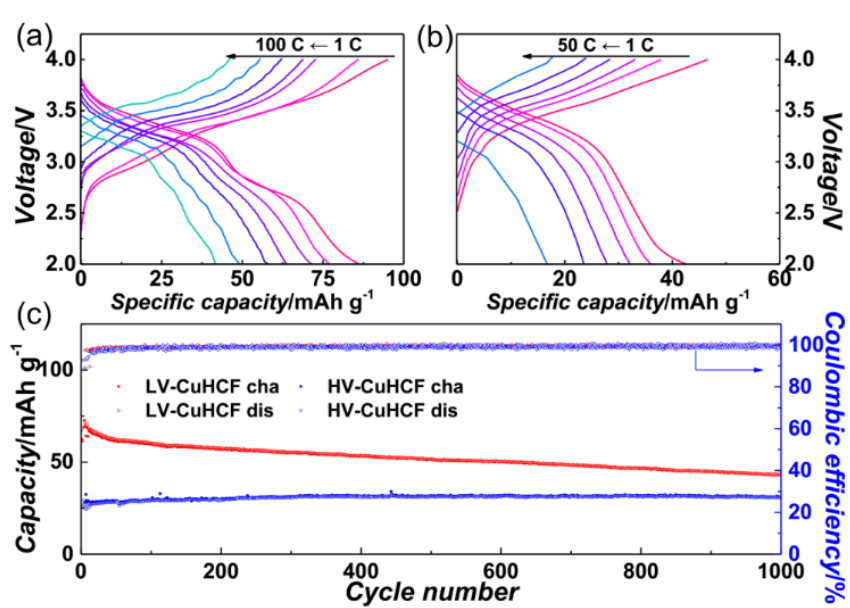

Figure 2. Rate performance of (a) LV-CuHCF (1 C to $100 \mathrm{C}$ ) and (b) HV-CuHCF $(1 \mathrm{C}$ to $50 \mathrm{C}$ ) at room temperature. (c) Long cycle performance of LV-CuHCF and HV-CuHCF. Rate: $10 \mathrm{C}$.

Such ultrafast kinetics featured by the outstanding rate capability is hard to be fulfilled, assuming the conventional diffusion-controlled electrochemical behaviors of electrode. Considering the three-dimensional open framework and the weak interaction of $\mathrm{Na}^{+}$with the anion $\mathrm{p}_{\pi}$ electrons, intercalation pseudocapacitance is predicted to play a crucial rule in realizing ultrahigh rate performance of LV-CuHCF. Cycling voltammetry (CV) analysis were carried out with variation of sweep rates $(0.1$ $300 \mathrm{mV} \mathrm{s}^{-1}$ ) to examine the intercalation behavior of $\mathrm{Na}^{+}$in the LV-CuHCF electrode (Figure $3 a$ and Figure S2). In accord with the two voltage plateaus in the charge/discharge profiles of LVCuHCF, two pairs of redox peaks are observed in the CV curves at low sweep rates (Figure S2a). Upon increasing sweep rate, there are peak shifts for both cathodic and anodic peaks, as well as the evanescence of the low-voltage peaks couple $(2.75 / 3.10$ $\mathrm{V})$. The remaining pair of peaks $(3.40 / 3.25 \mathrm{~V})$ corresponds to the redox couple of $\left[\mathrm{Fe}(\mathrm{CN})_{6}\right]^{4-} /\left[\mathrm{Fe}(\mathrm{CN})_{6}\right]^{3-}$, which is commonly observed in previous studies. In principle, the current response $(I)$ of $\mathrm{CV}$ scans can be described as a power-law formula with the sweep rate $(V)^{[11,20] \text { : }}$

$i=a v^{b}$

where $b$ reflects the control process of the electrochemical behavior. The variation of $b$-value from 0.5 to 1 represents the transformation of the electrochemical behavior from a diffusioncontrolled process to a surface-controlled one. As shown in Figure $3 b$, for the sweep rates from 1 to $10 \mathrm{mV} \mathrm{s}^{-1}$, the $b$-values for the cathodic and anodic peaks are 0.85 and 0.88 , respectively, suggesting that the current is primarily surface-controlled in LVCuHCF. Specifically as shown in Figure S3, the capacitive contribution gradually increases from $63 \%$ to $84 \%$ upon increasing the sweep rate from 0.5 to $4 \mathrm{mV} \mathrm{s}^{-1}$, further verifying the domination of capacitive storage to the total electrochemical process. When increasing the sweep rate to $20 \mathrm{mV} \mathrm{s}^{-1}$ and above, the $b$-values decreases to 0.54 and 0.62 for the cathodic and anodic peaks respectively, indicating a slow diffusion-controlled process in the electrode. Such phenomenon is associated with an increase of the ohmic contribution and/or diffusion constraints/limitations under ultrahigh sweep rates, which has been also reported in other intercalation electrodes such as $\mathrm{TiO}_{2}$ and $T-\mathrm{Nb}_{2} \mathrm{O}_{5} \cdot{ }^{[11,21]}$
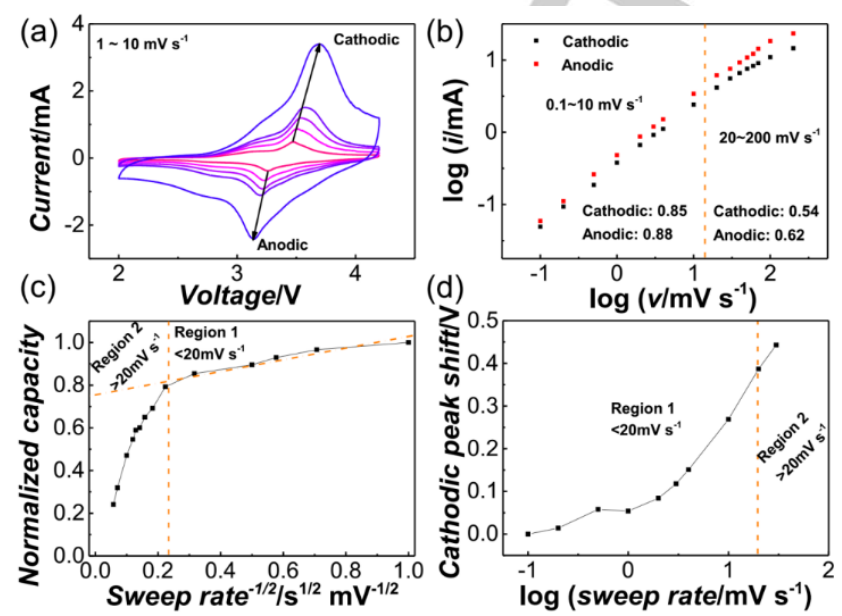

Figure 3. Kinetics analysis of sodium-ion intercalation in LV-CuHCF. a) CVs of LV-CuHCF at various scan rates $(v)$ from 1 to $10 \mathrm{mV} \mathrm{s}^{-1}$. b) $\log (i)$ versus log $(v)$ curves of cathodic and anodic peaks. C) Normalized capacity versus $V^{1 / 2}$ curves at scan rates of $0.1-300 \mathrm{mV} \mathrm{s}^{-1}$. The orange cater-corner in this figure is the extrapolation between 10 and $20 \mathrm{mV} \mathrm{s}^{-1}$. d) Curves of $\log (v)$ versus cathodic peak potential shifts.

In addition, the rate-limiting step of LV-CuHCF electrode can be further identified by the relationship between capacity and sweep rate. Since solid-state diffusion always exhibits linear dependence of sweep rate in a plot of normalized capacity versus $v^{1 / 2}$, a capacity roughly independent of sweep rate from 0.1 to $10 \mathrm{mV} \mathrm{s}$ ${ }^{1}$ (region 1 shown in Figure 3c) manifests that the rate-limiting process in our LV-CuHCF electrode is surface-controlled. In region 2 , from 20 to $300 \mathrm{mV} \mathrm{s}^{-1}$, the capacity decreasing linearly with $v^{1 / 2}$ indicates that the rate-limiting step changes from surface-controlled to diffusion-controlled at high sweep rates. That is, diffusion is rate-limiting for charging time of less than 60 $\mathrm{s}$, similar to most conventional electrodes. However, for charging period above $120 \mathrm{~s}(\approx 16 \mathrm{C})$, a fully capacitive manner dominates the behavior of this intercalation-based system with no indication of diffusion limitations. Another feature identifying the intercalation pseudocapacitance behavior of LV-CuHCF is the small peak shift with sweep rate. As shown in Figure $3 \mathrm{~d}$, the cathodic peak shift is less than $0.2 \mathrm{~V}$ at sweep rate below $5 \mathrm{mV} \mathrm{s}^{-1}$. Besides, despite a little higher charge transfer resistance $\left(R_{c t}\right)$ (Figure $\left.S 4 a\right)$, sodium diffusion coefficient $\left(D_{\mathrm{Na}+}\right)$ of LV-CuHCF calculated from the relationship between $Z_{\text {re }}$ and $\omega^{-1 / 2}$ in low frequency (Figure $S 4 b$ ) is $5.7 \times 10^{-11} \mathrm{~cm}^{2} \mathrm{~s}^{-1}$, an order of magnitude higher than that of HVCuHCF $\left(5.44 \times 10^{-12} \mathrm{~cm}^{2} \mathrm{~s}^{-1}\right)$, indicating the fast diffusion process. ${ }^{[12,22]}$

The above-mentioned redox reaction of $\mathrm{Cu}^{+} / \mathrm{Cu}^{2+}$ which contributes considerable capacity in the LV-CuHCF electrode is verified by ex-situ X-ray photoelectron spectroscopy (XPS) and ex-situ XRD at different states of charge (Figure 4). As displayed in Figure $4 \mathrm{a}$, the existence of $\mathrm{Cu}(\mathrm{II})$ is identified in the electrode 
at a charged state $(3.4 \mathrm{~V})$. With an increasing amount of inserted $\mathrm{Na}^{+}$ions in the framework, the peak at $933.2 \mathrm{eV}$ shifts to lower binding energy. In the meantime, the peak of $\mathrm{Cu}(\mathrm{I})$ appears till the electrode is discharged to $2.0 \mathrm{~V}$. The transformation from cupric to cuprous in CuHCF has been demonstrated by Giorgetti et al. through X-ray absorption spectroscopy (XAS)..$^{[23]}$ Nevertheless, negligible capacity is contributed by this less activated $\mathrm{Cu}^{+} / \mathrm{Cu}^{2+}$ redox couple due to the insufficient ionic and electronic conductivity in previously reported CuHCF electrodes. ${ }^{[14]}$

Meanwhile, ex-situ XRD analysis (Figure 4c) shows the phase variation of LV-CuHCF at different states of charge (Figure 4b). The XRD peaks shift negligibly during the $\mathrm{Na}^{+}$extraction/insertion process with a volume change less than 1\% (See Supporting Information), demonstrating the robust structure of LV-CuHCF, which is a key factor in achieving intercalation pseudocapacitance. Noteworthy, the XRD peaks shift gradually toward higher angle during $\mathrm{Na}$ extraction and oppositely change during $\mathrm{Na}$ insertion except at the charge state of $2.0 \mathrm{~V}$. The abnormal volume shrinkage upon charging from $2.0 \mathrm{~V}$ to $2.8 \mathrm{~V}$ may be caused by the Jahn-Teller effect of $\mathrm{Cu}^{2+}$ that leads to strong distortion of $\mathrm{CuN}_{6}$ octahedra. The twist of $\mathrm{CuN}_{6}$ octahedra will be generated by the oxidation of $\mathrm{Cu}^{+}$upon sodium insertion. On the contrary, such structure transformation is not observed in HV-CuHCF electrode, as displayed in Figure S5. Such discrepancy between LV-CuHCF and HV-CuHCF further verifies the redox activity of $\mathrm{Cu}$ in LV-CuHCF.

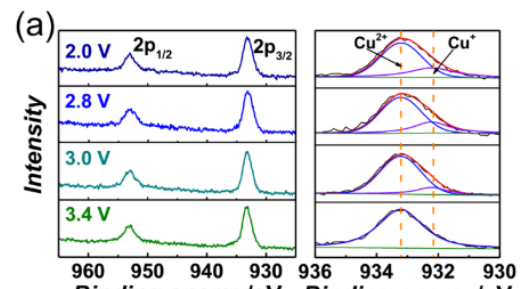

$$
\text { (b) }
$$

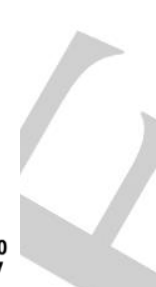
(C)

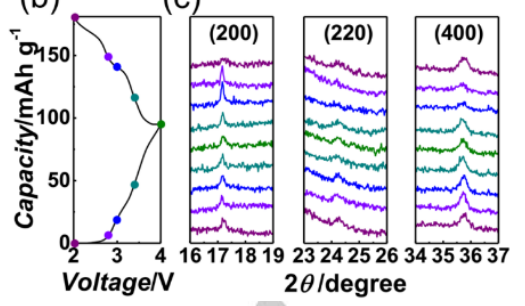
$2 \theta /$ degree

Figure 4. (a) ex-situ XPS spectrum of LV-CuHCF at various states of charge. (b) A typical charge-discharge profile of LV-CuHCF electrode at $1 \mathrm{C}$ rate and (c) $e x$ situ XRD patterns corresponding to different states of charge in (b). The (200) peak of LV-CuHCF at different charge-discharge states is $17.169^{\circ}, 17.144^{\circ}$, $17.177^{\circ}, 17.179^{\circ}, 17.199^{\circ}, 17.180^{\circ}, 17.162^{\circ}, 17.159^{\circ}, 17.163^{\circ}$, correspondingly.

As applied to ESSs, fast charge/discharge with superior cycling performance is required not only at room temperature, but also at higher and lower temperatures. Since low temperature decreases the energy and power densities of the cathodes more significantly due to the sluggish solid-diffusion process, the need for cathode materials with ultrahigh rate capability is even more urgent. LVCuHCF with intercalation pseudocapacitive behavior may satisfy

the requirements for application at low temperature. Galvanostatic charge-discharge profiles of LV-CuHCF and HVCuHCF in half-cells under different rates at $0{ }^{\circ} \mathrm{C}$ are shown in Figure $5 \mathrm{a}$ and $5 \mathrm{~b}$, respectively. The LV-CuHCF electrode delivers a capacity of $61,55,46,38,27$, and $19 \mathrm{mAh} \mathrm{g}^{-1}$ at 1, 2, 5, 10, 20 and $40 \mathrm{C}$ rates. Opposite to HV-CuHCF that delivers a specific capacity of only $26 \mathrm{mAh} \mathrm{g}^{-1}$ at $1 \mathrm{C}$ and nearly no capacity upon the rate increasing to $40 \mathrm{C}, \mathrm{LV}$-CuHCF maintains impressive rate capabilities even at freezing point. In term of cycling stability, LVCuHCF maintains a reversible capacity as high as $60 \mathrm{mAh} \mathrm{g}^{-1}$ after 100 cycles with negligible capacity fading under $1 \mathrm{C}$ at $0{ }^{\circ} \mathrm{C}$ while HV-CuHCF only delivers less than a half of the capacity of LV-CuHCF, as displayed in Figure 5c. The better cycle stability may be twofold. One reason is the weaker thermal vibration at lower temperature reduces the possibility of structure collapse during cycling. Another reason may be the reduction of side reactions at low temperature.

More details can be seen in their charge-discharge profiles shown in Figure S6a. The plateau corresponding to the $\left[\mathrm{Fe}(\mathrm{CN})_{6}\right]^{4-/}\left[\mathrm{Fe}(\mathrm{CN})_{6}\right]^{3-}$ couple in LV-CuHCF is much longer than that of HV-CuHCF and the plateau corresponding to $\mathrm{Cu}^{+} / \mathrm{Cu}^{2+}$ couple in LV-CuHCF can still be observed clearly. The typical shape of the discharge curve of LV-CuHCF can remain even cycling under a tenfold rate at $0^{\circ} \mathrm{C}$, while the curve of $\mathrm{HV}$-CuHCF becomes a straight line with negligible capacity under the same condition (Figure S6b). The capacity retention of LV-CuHCF and HV-CuHCF at $0{ }^{\circ} \mathrm{C}$ calculated on the basis of their own specific capacities under different current rates at room temperature is exhibited in Figure 5d. Fairly high capacity retention above $70 \%$ is achieved by LV-CuHCF at both 1 and $2 \mathrm{C}$ rates. $10 \%$ more capacity retention can be obtained in the LV-CuHCF electrode than that in the HV-CuHCF electrode at various current rates from $1 \mathrm{C}$ to $20 \mathrm{C}$. Apart from the fast ion diffusion, LV-CuHCF also possess the fast charge transfer kinetics at $0{ }^{\circ} \mathrm{C}$ (Figure S7). The above factors assuage the polarization of LV-CuHCF at low temperature synergistically, resulting in a high capacity even at high current rates.

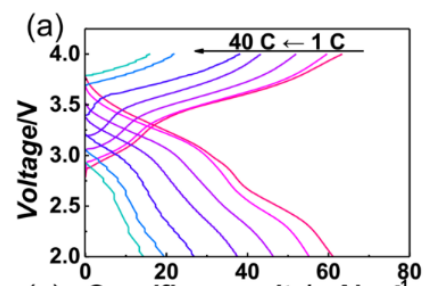

(c) Specific capacity/mAh g g $^{-1}$ (d) Specific capacity/mAh g g $^{-1}$
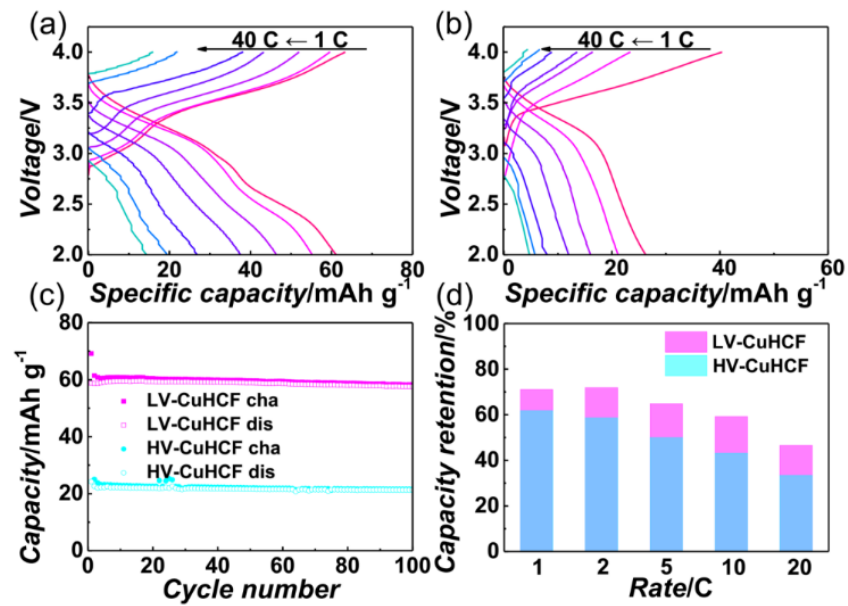

Figure 5. Rate performance of (a) LV-CuHCF and (b) HV-CuHCF from $1 \mathrm{C}$ to $40 \mathrm{C}$ at $0^{\circ} \mathrm{C}$. (c) Cycle performance of LV-CuHCF and HV-CuHCF at $0^{\circ} \mathrm{C}$. Rate: 
1 C. (d) Capacity retention of LV-CuHCF and HV-CuHCF at $0{ }^{\circ} \mathrm{C}$ under same current densities.

\section{Conclusions}

In summary, LV-CuHCF was prepared in a dehydrated environment to form highly crystallized nanocubes, achieving outstanding rate performance and high reversible capacity toward sodium storage. A unique intercalation pseudocapacitance is demonstrated in LV-CuHCF due to the elimination of solid-state diffusion limits, benefitting from the unbroken sodium diffusion pathway and little structural change of Fe redox. At room temperature, the LV-CuHCF electrode can deliver a discharge capacity of $86 \mathrm{~mA} \mathrm{~h} \mathrm{~g}^{-1}$ at $1 \mathrm{C}$ and a capacity retention of $\sim 50 \%$ when the current rate increases to $100 \mathrm{C}$. The long cyle life is also demonstrated with a reversible capacity of $42 \mathrm{~mA} \mathrm{~h} \mathrm{~g}^{-1}$ at $10 \mathrm{C}$ over 1000 cycles. In addition, the minimization of $\left[\mathrm{Fe}(\mathrm{CN})_{6}\right]$ vacancy activates the redox activity of $\mathrm{Cu}$ that provides a considerable capacity in LV-CuHCF. The LV-CuHCF electrode also exhibits outstanding low-temperature electrochemical performance with $70 \%$ capacity retention as the testing temperature dropping to $0^{\circ} \mathrm{C}$ at $1 \mathrm{C}$, and negligible capacity decay can be observed during cycling.

\section{Experimental Section}

Materials preparation: Both LV-CuHCF and HV-CuHCF were prepared by a co-precipitation method using $\mathrm{Na}_{4} \mathrm{Fe}(\mathrm{CN})_{6}$ and $\mathrm{CuCl}_{2}$ as precursors. For the preparation of HV-CuCHF, Ketjen black powder was first added in 100 $\mathrm{mL}$ deionized water followed by 1 hour ultrasonic pre-treatment. A $100 \mathrm{~mL}$ aqueous solution containing $\mathrm{CuCl}_{2}(20 \mathrm{mM})$ and a $100 \mathrm{~mL}$ aqueous solution of $\mathrm{Na}_{4} \mathrm{Fe}(\mathrm{CN})_{6}(20 \mathrm{mM})$ were separately dropwise added into the pre-prepared Ketjen black water under magnetic stirring using a peristaltic pump at $10 \mathrm{~mL} \mathrm{~h}^{-1}$. The mixture was maintained at $90^{\circ} \mathrm{C}$ for $10 \mathrm{~h}$ under vigorous stirring to obtain the HV-CuHCF. For LV-CuHCF, Ketjen black powder was added in a solution of $75 \mathrm{~mL}$ deionized water and $25 \mathrm{~mL}$ ethanol. For the $\mathrm{CuCl}_{2}$ precursor, $\mathrm{CuCl}_{2} \cdot 4 \mathrm{H}_{2} \mathrm{O}$ was heated at $120^{\circ} \mathrm{C}$ for 10 minutes to remove the crystal water, and then dissolved in $100 \mathrm{~mL}$ ethanol. Then the ethanol solution and a $100 \mathrm{~mL}$ aqueous solution of $\mathrm{Na}_{4} \mathrm{Fe}(\mathrm{CN})_{6}$ $(20 \mathrm{mM})$ were separately dropwise added into the pre-prepared Ketjen black dispersion under the same condition of HV-CuHCF. The mixture was maintained at $90^{\circ} \mathrm{C}$ for $10 \mathrm{~h}$ under vigorous stirring to obtain the LVCuHCF. The two composites were collected by filtration, washed by deionized water for three times, and dried at $120^{\circ} \mathrm{C}$ in a vacuum oven for $48 \mathrm{~h}$.

Structure and morphology characterization: The identification of the crystalline phase was performed using X-ray diffraction (XRD) on a Rigaku D/Max2550pc diffractometer equipped with Cu Ka radiation $(\lambda=1.5406 \AA)$. The surface morphology was characterized by scanning electron microscopy (SEM, Hitachi S-4800). X-ray photoelectron spectra were recorded by an Escalab 250Xi XPS spectrometer using a monochromatized $\mathrm{Al} \mathrm{Ka}$ radiation with $40 \mathrm{eV}$ step over the sample. The chemical composition was examined by elemental analysis (Vario Micro) for $\mathrm{C}, \mathrm{N}$ and $\mathrm{H}$ elements, and by ICPAES (IRIS INTREPID II XSP) for Fe, $\mathrm{Cu}$ and Na elements. The formulae of LV-CuHCF and HV-CuHCF were $\mathrm{Na} \times \mathrm{Cu}\left[\mathrm{Fe}(\mathrm{CN})_{6}\right]_{0.931} \cdot \mathrm{nH}_{2} \mathrm{O}$ and $\mathrm{Na} \times \mathrm{Cu}\left[\mathrm{Fe}(\mathrm{CN})_{6}\right]_{0.647} \cdot \mathrm{nH}_{2} \mathrm{O}$, respectively.
Electrochemical test: For HV-CuHCF electrode, a slurry composed of HVCuHCF, Ketjen black and poly(vinyldifuoride) (PVDF) was coated onto aluminum foil to prepare the working electrode. Extra Ketjen black was added into the slurry to make sure the weight ratio of the active material, conductive carbon and the binder is 7:2:1. The electrode was dried in a vacuum oven at $120^{\circ} \mathrm{C}$ overnight. For LV-CuHCF electrode, the active material was replaced by HV-CuHCF. The typical electrode mass loading was $1-2 \mathrm{mg} \mathrm{cm}^{-2}$. The specific capacity was calculated based on the mass of the active materials. Electrochemical tests were carried out in a 2025 cointype half-cell, in which a piece of sodium foil was used as both the counter and reference electrode and $1.0 \mathrm{M} \mathrm{NaClO}_{4}$ in ethylene carbonate $(E C)$, diethyl carbonate (DEC) and propylene carbonate (PC) $(2: 2: 1$ in a volume ratio) was used as the electrolyte. The cells were assembled in an argon-filled glove box. Galvanostatic tests were carried out in the potential range of 2.0-4.0 V (vs. $\mathrm{Na}^{+} / \mathrm{Na}$ ) using a battery test system (Neware BTS-5) at room temperature and $0{ }^{\circ} \mathrm{C}$. Cyclic voltammetry (CV) tests were performed using an Ivium vertex $\mathrm{C}$ electrochemistry workstation. Electrochemical impedance spectra (EIS) were measured using a CHI660D electrochemistry workstation in the frequency range of $100 \mathrm{kHz}$ to $0.1 \mathrm{~Hz}$.

\section{Acknowledgements}

This study was supported by National key research and development program (Grant No. 2016YFB0901600), National Natural Science Foundation of China (Grant No. 51722105), Zhejiang Provincial Natural Science Foundation of China (LR18B030001), and the Fundamental Research Funds for the Central Universities (2018XZZX002-08).

Keywords: sodium ion batteries $\cdot$ cathodes - Prussian blue analogues $\bullet$ intercalation pseudocapacitance $\cdot$ rate capability

[1] a) H. Kim, H. Kim, Z. Ding, M. H. Lee, K. Lim, G. Yoon, K. Kang, Adv Energy Mater. 2016, 6, 1600943; b) P. K. Nayak, L. T. Yang, W. Brehm, P. Adelhelm, Angew. Chem. Int. Ed. 2018, 57, 102-120.

[2] C. Vaalma, D. Buchholz, M. Weil, S. Passerini, Nat. Rev. Mater. 2018, 3 18013.

[3] J. Y. Hwang, S. T. Myung, Y. K. Sun, Chem. Soc. Rev. 2017, 46, 35293614.

[4] Y. You, A. Manthiram, Adv. Energy Mater. 2018, 8, 1701785

[5] a) N. A. Katcho, J. Carrasco, D. Saurel, E. Gonzalo, M. Han, F. Aguesse, T. Rojo, Adv. Energy Mater. 2017, 7, 1601477; b) P. Wang, Y. You, Y. Yin, Y. Guo, J. Mate. Chem. A 2016, 4, 17660-17664.

[6] a) Y. Xu, S. Zheng, H. Tang, X. Guo, H. Xue, H. Pang, Energy Stor. Mater. 2017, 9, 11-30; b) A. Paolella, C. Faure, V. Timoshevskii, S. Marras, G Bertoni, A. Guerfi, A. Vijh, M. Armand, K. Zaghib, J. Mater. Chem. A 2017, 5, 18919-18932; c) J. Qian, C. Wu, Y. Cao, Z. Ma, Y. Huang, X. Ai, H. Yang, Adv. Energy Mater. 2018, 8, 1702619.

[7] a) P. Wang, Y. You, Y. Yin, Y. Guo, Adv. Energy Mater. 2018, 8, 1701912; b) P. Wang, H. Yao, X. Liu, J. Zhang, L. Gu, X. Yu, Y. Yin, Y Guo, Adv. Mater. 2017, 29, 1700210.

[8] a) W. Guan, B. Pan, P. Zhou, J. Mi, D. Zhang, J. Xu, Y. Jiang, ACS Appl. Mater. Interfaces 2017, 9, 22369-22377; b) W. Zhou, L. Xue, X. Lü, H. Gao, Y. Li, S. Xin, G. Fu, Z. Cui, Y. Zhu, J. B. Goodenough, Nano Lett. 2016, 16, 7836-7841; c) F. Chen, V. M. Kovrugin, R. David, O. Mentré F. Fauth, J.-N. Chotard, C. Masquelier, Small Meth. 2018, 1800218.

[9] X. Wu, W. Deng, J. Qian, Y. Cao, X. Ai, H. Yang, J. Mater. Chem. A 2013 1, 10130-10134

[10] Y. You, H. Yao, S. Xin, Y. Yin, T. Zuo, C. Yang, Y. Guo, Y. Cui, L. Wan, J. B. Goodenough, Adv. Mater. 2016, 28, 7243. 
[11] V. Augustyn, J. Come, M. A. Lowe, J. W. Kim, P. L. Taberna, S. H Tolbert, H. D. Abruna, P. Simon, B. Dunn, Nat. Mater. 2013, 12, 518-522.

[12] J. Ding, Z. Du, L. Gu, B. Li, L. Wang, S. Wang, Y. Gong, S. Yang, Adv. Mater. 2018, 30, 1800762.

[13] T. Brezesinski, J. Wang, S. H. Tolbert, B. Dunn, Nat. Mater. 2010, 9, 146151.

[14] a) S. Jiao, J. Tuo, H. Xie, Z. Cai, S. Wang, J. Zhu, Mater. Res. Bull. 2017, 86, 194; b) Z. Jia, J. Wang, Y. Wang, RSC. Adv. 2014, 4, 22768-22774.

[15] a) P. Jiang, H. Shao, L. Chen, J. Feng, Z. Liu, J. Mater. Chem. A 2017, 5, 16740-16747; b) D. J. Kim, Y. H. Jung, K. K. Bharathi, S. H. Je, D. K. Kim, A. Coskun, J. W. Choi, Adv. Energy Mater. 2014, 4, 1400133; c) C D. Wessells, M. T. McDowell, S. V. Peddada, M. Pasta, R. A. Huggins, Y. Cui, Acs Nano 2012, 6, 1688-1694; d) M. Pasta, C. D. Wessells, N. Liu, J. Nelson, M. T. McDowell, R. A. Huggins, M. F. Toney, Y. Cui, Nat. Comm. 2014, 5, 3007.

[16] C. D. Wessells, R. A. Huggins, Y. Cui, Nat. Comm. 2011, 2, 550.
[17] Y. You, X. Wu, Y. Yin, Y. Guo, Energy Environ. Sci. 2014, 7, 1643-1647.

[18] D. Yang, J. Xu, X. Z. Liao, H. Wang, Y. S. He, Z. F. Ma, Chem. Comm 2015, 51, 8181-8184.

[19] a) Y. Huang, M. Xie, J. Zhang, Z. Wang, Y. Jiang, G. Xiao, S. Li, L. Li, F. Wu, R. Chen, Nano Energy 2017, 39, 273-283; b) B. Wang, Y. Han, X. Wang, N. Bahlawane, H. Pan, M. Yan, Y. Jiang, iScience 2018, 3, 110133.

[20] Y. Jiang, Y. Li, P. Zhou, Z. Lan, Y. Lu, C. Wu, M. Yan, Adv. Mater. 2017 29, 1606499.

[21] V. Augustyn, E. R. White, J. Ko, G. Gruner, B. C. Regan, B. Dunn, Mater Horizons 2014, 1, 219-223.

[22] S. B. Yang, Y. J. Gong, Z. Liu, L. Zhan, D. P. Hashim, L. L. Ma, R. Vajtai, P. M. Ajayan, Nano Lett. 2013, 13, 1596-1601.

[23] A. Mullaliu, G. Aquilanti, P. Conti, J. R. Plaisier, M. Fehse, L. Stievano, M. Giorgetti, J. Phys. Chem. C 2018, 122, 15868-15877. 


\section{Entry for the Table of Contents}

\section{FULL PAPER}

Intercalation pseudocapacitance is demonstrated in Prussian blue analogue toward sodium storage of copper hexacyanoferrate for the first time. The minimization of $\left[\mathrm{Fe}(\mathrm{CN})_{6}\right]$ vacancy in copper hexacyanoferrate enables unhindered diffusion pathway for $\mathrm{Na}^{+}$and little structural change during $\mathrm{Fe}^{2+} / \mathrm{Fe}^{3+}$ redox reaction, eliminating solid-state diffusion limits. High reversible capacity, outstanding rate capability and long cycle life are achieved simultaneously.
Baoqi Wang, Shuangyu Liu, Wenping
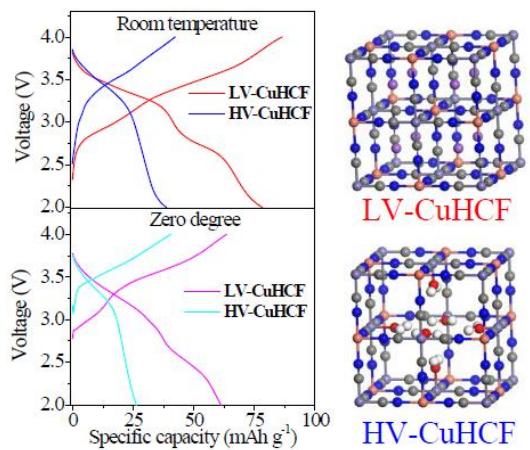
Sun, Yuxin, Tang, Hongge Pan, Mi Yan, Yinzhu Jiang*

\section{Page No. - Page No.}

Intercalation pseudocapacitance boosted sodium storage in Prussian blue analogues 